\title{
Support for disease management, depression, self-care, and clinical indicators among Hispanics with type 2 diabetes in San Diego County, United States of America
}

\author{
Addie L. Fortmann, ${ }^{1}$ Linda C. Gallo, ${ }^{1}$ Chris Walker, ${ }^{2}$ \\ and Athena Philis-Tsimikas ${ }^{2}$
}

Suggested citation

Fortmann AL, Gallo LC, Walker C, Philis-Tsimikas A. Support for disease management, depression, self-care, and clinical indicators among Hispanics with type 2 diabetes in San Diego County, United States of America. Rev Panam Salud Publica. 2010;28(3):230-4.
ABSTRACT This study used a social-ecological framework to examine predictors of depression, diabetes self- management, and clinical indicators of health risk among Hispanics with type 2 diabetes resid- ing in the United States (U.S.)-Mexico border region in San Diego County, California, United States of America. Important links were observed between greater social-environmental support for disease management and less depression, better diabetes self-management, and lower body mass index and serum triglyceride concentrations. Less depressive symptomatol- ogy was also related to lower hemoglobin A1c levels. Findings suggest that programs aiming to improve diabetes self-management and health outcomes in Hispanics with type 2 diabetes should consider multilevel, social, and environmental influences on health, behavior, and emo- tional well-being.

Key words Hispanic Americans; border health; diabetes mellitus, type 2; disease management; depression.

Diabetes mellitus, one of the fastest growing chronic illnesses in the United States of America, refers to a group of metabolic diseases characterized by abnormally high blood glucose levels. In 2008, the Centers for Disease Control and Prevention reported that 24 million people in the United States were diagnosed with diabetes and estimated that another 57 million people met criteria for prediabetes, a condition that puts people at greater risk for developing diabetes.

\footnotetext{
1 San Diego State University/University of California, San Diego, Joint Doctoral Program in Clinical Psychology, San Diego, California, United States of America. Send correspondence to Addie L. Fortmann, abrewer@projects.sdsu. edu

2 Scripps Whittier Diabetes Institute, La Jolla, California, United States of America.
}

Type 2 diabetes, in particular, occurs when the pancreas creates insufficient amounts of insulin or when the body becomes unable to use its limited supply of insulin effectively.

The National Institutes of Health defines health disparities as the "differences in the incidence, prevalence, mortality and burden of disease and other adverse health conditions that exist among specific population groups in the United States." Type 2 diabetes, among some other chronic conditions, is unequally distributed across ethnic groups. In particular, the Hispanic population exhibits a higher prevalence of type 2 diabetes, more frequent complications, greater disease severity, and worse out- comes than non-Hispanic whites $(1,2)$ The U.S.-Mexico border region, in particular, is marked by significant health disparities. More than 12 million people reside in states bordering Mexico, and most live in medically underserved areas. Rapid population growth and industrialization, inadequate housing and roads, sewage problems, unsafe drinking water, poverty, and language barriers contribute to the health issues that confront border residents and pose challenges to their seeking care.

The burden of chronic diseases such as diabetes in Hispanics living along the U.S.-Mexico border is salient, and prevalence levels are rising. A 2004 report by the Pan American Health Organization 
(PAHO) showed that individuals residing in the border region exhibited close to a $16 \%$ prevalence of type 2 diabetes. Further, the prevalence of diabetes among Hispanics in this region is 2 to 2.5 times greater than in non-Hispanic whites in the same area (3). Depression and inadequate diabetes self-management are common among Hispanics with type 2 diabetes $(4,5)$ and represent two potential reasons why this population experiences worse diabetes-related health outcomes than non-Hispanic whites.

Depression is two times more prevalent among persons with type 2 diabetes than in the general population and is more common among Hispanics than among blacks and non-Hispanic whites with the same condition (5). Prevalence rates are especially high along the U.S.-Mexico border (e.g., 39\% in south Texas and $40.5 \%$ in northern Mexico) (6). The interaction between depression and diabetes intensifies the adverse effects of both conditions, resulting in increased use of healthcare services, more frequent and lengthier hospitalizations, and greater costs associated with care (2). Among individuals with type 2 diabetes, depression is associated with poorer compliance with treatment recommendations, worse glycemic control, increased diabetes complications, and higher mortality rates. Accordingly, depression is an important factor to consider when investigating and addressing diabetes health disparities in this population.

Individuals with uncontrolled diabetes are at risk for developing serious health complications, including hypertension, dyslipidemia, cardiovascular disease, retinopathy, nephropathy, and neuropathy. Fortunately, the development and progression of serious health consequences can be delayed or prevented through diabetes self-management techniques that promote tight glucose control. Diabetes self-management involves daily engagement in a variety of behaviors, including healthy eating, physical activity, blood glucose monitoring, taking medications, problem solving, and adaptive coping.

Although active diabetes selfmanagement is required to achieve glycemic control and prevent serious health complications, adherence in the Hispanic population is lacking. For instance, data from the Behavioral Risk Factor Surveillance System found that only $59.7 \%$ of respondents in the Texas U.S.-Mexico border region had their hemoglobin A1c levels (HbA1c) checked at least once annually. Research has also shown that when Hispanics do seek healthcare services, they receive a poorer quality of care than non-Hispanic whites when treated for the same condition (4). Hispanics living on the U.S. side of the border often receive most of their healthcare services in Mexico because of lower costs and easier access.

Several potential explanations have been put forth to explain why Hispanics with type 2 diabetes exhibit higher rates of depression and poorer diabetes selfmanagement than non-Hispanic whites with the same condition. Lower socioeconomic status, limited access to healthcare, comorbid medical conditions, and cultural and language barriers pose unique health challenges to this population. For instance, nearly $20 \%$ of Hispanics do not seek care because of language barriers, and more than $80 \%$ of Hispanic patients over the age of 60 years cannot read or understand health-related materials (4). Research conducted in the U.S.-Mexico border region identified "lack of money" as a commonly reported barrier to adherence (4).

Additionally, perceived difficulty of self-management behaviors and a perceived lack of control over one's health may affect emotional well-being and lower motivation to comply with self-care regimens. Hispanics, in particular, perceive self-monitoring of blood glucose as more difficult and have a poorer outlook on diabetes than Asians, African Americans, and non-Hispanic whites (7). Sociocultural factors may also play an important role in depression and diabetes self-management in this population.

Hispanics place great value on personal relationships (i.e., personalismo), family (i.e., familismo), and community and are especially dependent on their social networks during times of need (e.g., chronic illness) (8). Previous research has identified social support as a protective factor in the development of depression among individuals with chronic illness. More specifically, studies have shown that individuals with type 2 diabetes who report receiving more social support are less likely to exhibit comorbid depression than those with relatively less support $(9,10)$. The sociocultural context may also be linked to diabetes self-management in the Hispanic population. For instance, research has shown family cohesion to be an important motivator for diabetes self-management in this population; family members provided assistance by monitoring, advising, and directing adherence to diabetes self-management behaviors (11).

Previous research has shown that depression and poor diabetes selfmanagement contribute, at least in part, to the poorer health outcomes experienced by Hispanics. Less is known, however, about the sociocultural factors that influence depression and diabetes selfmanagement in this population. As such, the present correlational study investigated support for disease management (defined here as social-environmental support and resources for disease management from proximal and distal sources) as a predictor of depression, diabetes self-management, and ultimately clinical indicators of health risk in a sample of Hispanics with type 2 diabetes residing in the U.S.-Mexico border region.

Specifically, it was hypothesized that individuals who report receiving greater support for disease management would endorse less depressive symptomatology, report better diabetes self-management, and exhibit more optimal values on clinical indicators-i.e., lower $\mathrm{HbA1c}$, total cholesterol, low density lipoprotein cholesterol (LDL-c), serum triglyceride concentrations, blood pressure, and body mass index (BMI), and higher high density lipoprotein cholesterol (HDL-c) than individuals reporting relatively less support. Relationships between depression and each of the physiological indicators were also investigated.

Hispanic men and women between 21 and 75 years old with a diagnosis of type 2 diabetes (confirmed via medical chart review) and $\mathrm{HbA1c}$ greater than $8 \%$ were recruited between July 2007 and March 2009 from low-income-serving community clinics in the U.S.-Mexico border region of San Diego County, California, USA. To be eligible for the study, participants had to be a MediCal enrollee or uninsured and enrolled in the community clinic. Individuals with a severe medical condition or poor level of mental functioning that would preclude frequent visits to the clinic or interfere with the requirements of the study were ineligible to participate.

Once enrolled, participants were randomly assigned to the treatment group or the control group. Those in the treat- 
ment group attended a class once a week for 8 weeks on managing diabetes (Project Dulce); they also participated in support groups led by trained promotoras. Individuals in the control group received standard care alone. All participants completed psychosocial assessments and underwent a physical assessment with a fasting venous blood draw at baseline and 4 and 10 months after the baseline measurement; however, only data collected at the baseline assessment are included in this study. Assessments were performed by a trained, bilingual clinical trials assistant at the clinical study sites.

Clinical indicators assessed at each time point included $\mathrm{HbA1c}$ (i.e., an integrated marker of blood glucose control, where higher levels indicate worse control), total cholesterol, LDL-c and HDL-c, serum triglycerides, systolic and diastolic blood pressures, and BMI. Support for disease management was measured using 10 items from the Chronic Illness Resources Survey (12), a scale designed to assess supports and resources according to the social-ecological perspective on self-management. Participants reported, on a five-point scale (from "not at all" to "a great deal"), the amount of support received from a variety of proximal (e.g., friends, family) and distal (e.g., community) sources. Responses were scored and averaged to create three subscales reflecting support received from family and friends, from within (i.e., self), and from the community; an overall support for disease management summary score was created by averaging all subscales. Diabetes self-management was measured with six items from the Summary of Diabetes Self-Care Activities scale (13), which assessed the number of days in the last week they had completed a variety of diabetes self-management behaviors (i.e., blood-glucose monitoring, diet, and exercise). Responses to items were averaged to create a diabetes self-management summary score. Participants also completed the PHQ-9 (14), a widely used measure of depressive symptoms in primary care and other populations. Items were scored and summed to create a depression score for each respondent. Participants also self-reported gender, age, country of origin, marital status, educational attainment (according to 20 categories ranging from no education to a doctoral-level degree), monthly income (according to 17 categories, ranging from
$<$ US\$ 500 to $\geq$ US\$ 8000 ), and whether they had health insurance.

All analyses were performed using the Statistical Package for Social Science (SPSS) software (version 17.0). Basic descriptive statistics were obtained to describe the age, gender, origin, income, education, and insurance status of participants. Model residuals were plotted and examined and transformations were performed on variables exhibiting substantial deviations from normality. Although these transformations substantially improved the distributions, there were no appreciable differences between results of analyses using transformed versus untransformed variables. Thus, for brevity and ease of interpretation, results are presented for untransformed data only. Bivariate correlation analyses were conducted to explore baseline associations between support for disease management, diabetes self-management, depression, and clinical indicators of health risk. Age was entered as a covariate in all analyses.

The 206 participants who completed the baseline assessment ranged in age from 21 to 75 years $($ mean $=50.62$, standard deviation $=10.90$ ); the majority was female, born in Mexico, and uninsured (see Table 1). Support for disease management subscale means ranged from 2.32 (neighborhood/community support subscale) to 3.54 (self-support subscale) out of a possible 5.00 (see Table 2). After controlling for age, greater self-support $(-0.22, P<0.01)$ and neighborhood/ community support $(r=-0.20, P<0.01)$ for disease management were linked to less depressive symptomatology. Participants who reported receiving greater support for disease management from their neighborhood and community, from family and friends, and from within (i.e., self-support) reported better diabetes self-management $(r=0.38$ to $0.41, P<0.01)$ than individuals reporting relatively less support (see Table 3 ). With regard to clinical indicators of health risk, individuals who reported receiving more support for disease management from their neighborhood and community exhibited lower BMI $(r=-0.17, P<$ $0.05)$ and serum triglycerides $(r=-0.14$, $P<0.05)$ than those with less support. Depression exhibited a significant relationship with only one clinical indicator, HbA1c $(r=0.20, P<0.01)$, indicating that participants who endorsed fewer depressive symptoms exhibited better
TABLE 1. Demographic characteristics of 206 Hispanics residing in the U.S.-Mexico border region in San Diego, California, United States, March 2007-July 2009

\begin{tabular}{lrr}
\hline \multicolumn{1}{c}{ Characteristic } & No. & $\%$ \\
\hline Gender & & \\
$\quad$ Female & 146 & 70.9 \\
$\quad$ Male & 60 & 29.1 \\
Marital status & & \\
$\quad$ Married & 102 & 49.5 \\
$\quad$ Unmarried & 104 & 50.5 \\
Country of origin & & \\
$\quad$ Mexico & 181 & 87.8 \\
$\quad$ United States & 17 & 8.3 \\
$\quad$ Other & 8 & 3.9 \\
Education & & \\
$\quad<8$ th grade & 108 & 52.0 \\
$\quad$ 28th grade & 98 & 48.0 \\
Insurance coverage & & \\
$\quad$ Insured & 70 & 34.0 \\
$\quad$ Uninsured & 136 & 66.0 \\
Household monthly & & \\
income \\
$\quad<1$ (US $\$$ ) & & \\
$\quad$ 1 001-1 999 & 103 & 50.0 \\
$\quad \geq 2$ 000 & 78 & 37.9 \\
a Income categories are collapsed for descriptive purposes.
\end{tabular}

glycemic control than those who reported relatively more depression.

Substantial research has shown that Hispanics exhibit a greater prevalence of type 2 diabetes as well as poorer glycemic control (15), more frequent complications, greater disease severity, and worse health outcomes than nonHispanic whites $(1,2)$. According to PAHO, health disparities are especially marked in the U.S.-Mexico border region. Previous research has shown that depression and poor diabetes selfmanagement contribute, at least in part, to the poorer health outcomes experienced by Hispanics. Less is known, however, about the sociocultural factors that influence depression and diabetes selfmanagement in this population.

This study investigated the associations of support for disease management with depression, diabetes selfmanagement, and clinical indicators of health risk among Hispanic men and women with type 2 diabetes residing along the U.S.-Mexico border in San Diego County, California. Individuals who reported greater self and neighborhood/community support endorsed less depressive symptomatology, while support for disease management from both proximal (i.e., self, family and friends) and more distal (i.e., neighborhood/ community) sources was associated with better diabetes self-management. 
TABLE 2. Descriptive statistics for psychosocial variables and clinical indicators in the study of Hispanics residing in the U.S.-Mexico border region in San Diego, California, United States, March 2007-July 2009

\begin{tabular}{lrrrr}
\hline \multicolumn{1}{c}{ Variable/lndicator } & Mean & SD $^{\mathrm{a}}$ & Minimum & Maximum \\
\hline Self-report variables & & & & \\
Support for disease management (total) & 2.78 & 1.02 & 1.00 & 5.00 \\
$\quad$ Family/friends subscale & 2.49 & 1.32 & 1.00 & 5.00 \\
$\quad$ Neighborhood/community subscale & 2.32 & 1.03 & 1.00 & 5.00 \\
$\quad$ Self-support subscale & 3.54 & 1.24 & 1.00 & 5.00 \\
$\quad$ Depression & 5.99 & 5.46 & 0.00 & 23.00 \\
$\quad$ Diabetes self-management & 3.17 & 1.39 & 0.33 & 7.00 \\
Clinical indicators of health risk & & & & \\
HbA1c (\%) & 10.4 & 1.7 & 7.9 & 15.3 \\
Cholesterol (mg/dL) & 195.37 & 49.33 & 106.00 & 361.00 \\
$\quad$ Total & 45.43 & 11.36 & 24.00 & 84.00 \\
$\quad$ HDL-c & 109.32 & 40.42 & 10.00 & 266.00 \\
$\quad$ LDL-c & 202.37 & 103.84 & 47.00 & 525.00 \\
Triglycerides (mg/dL) & & & & \\
Blood pressure (mm Hg) & 122.42 & 16.55 & 90.00 & 209.00 \\
$\quad$ Systolic & 74.89 & 7.80 & 54.00 & 91.00 \\
$\quad$ Diastolic & 31.53 & 6.13 & 23.00 & 53.00 \\
Body mass index & & & & \\
\hline
\end{tabular}

a SD: standard deviation.

${ }^{b} \mathrm{HbA1c}$ : glycosylated hemoglobin. The minimum observed value for $\mathrm{HbA1c}$ was $7.9 \%$, because an exception to the inclusion criterion of $\mathrm{HbA} 1 \mathrm{c}>8 \%$ was made for one patient whose $\mathrm{HbA} 1 \mathrm{c}$ exceeded $8 \%$ at screening and enrollment but decreased to $7.9 \%$ by the baseline assessment.

${ }^{c}$ HDL-c: high density lipoprotein cholesterol.

d LDL-c: low density lipoprotein cholesterol.

TABLE 3. Baseline associations ${ }^{a}$ of support for disease management with depression and diabetes self-management among Hispanics residing in the U.S.-Mexico border region in San Diego, California, United States, March 2007-July 2009

\begin{tabular}{lcc}
\hline & \multicolumn{2}{c}{ Correlation coefficient $(r)$} \\
\cline { 2 - 3 } $\begin{array}{c}\text { Source of support for } \\
\text { disease management }\end{array}$ & $\begin{array}{c}\text { Diabetes self- } \\
\text { management }\end{array}$ & Depression \\
\hline Family and friends & $0.40^{\mathrm{b}}$ & -0.07 \\
Neighborhood and community & $0.38^{\mathrm{b}}$ & $-0.20^{\mathrm{b}}$ \\
Self & $0.41^{\mathrm{b}}$ & $-0.22^{\mathrm{b}}$ \\
Overall (all sources combined) & $0.45^{\mathrm{b}}$ & $-0.18^{\mathrm{c}}$ \\
\hline
\end{tabular}

${ }^{a}$ Age was included as a covariate in all analyses.

b $P<0.01$

c $P<0.05$.

Neighborhood/community support for disease management was the only support subscale associated with clinical indicators. Specifically, lower BMI and serum triglycerides were observed among participants who reported greater support from their neighborhood and community. Consistent with previous research, less depression was associated with better glycemic control (i.e., lower HbA1c).

Individuals with type 2 diabetes who experience comorbid depression manage their diabetes less effectively, are less socially and physically active, and report less life satisfaction and hope about the future than nondepressed individuals (2). However, the present findings highlight support for disease management as a potential protective factor in the development of depression among Hispanics with type 2 diabetes. Consistent with previous research, less depressive symptomatology was also linked to better glycemic control in this sample (i.e., lower $\mathrm{HbA} 1 \mathrm{c})$. Considering the adverse impact of depression on glycemic control and diabetes-related health outcomes (2), the link between support for disease management and depression is important; it represents a potential avenue by which depression, and thus adverse health outcomes, can be prevented, reduced, or eliminated.

Adherence to a diabetes self-care regimen is a prerequisite for achieving tight glycemic control and delaying or preventing the development and pro- gression of serious health consequences. Traditional approaches view diabetes self-management as the responsibility of the individual; however, this perspective is incongruent with cultural values held by many Hispanics. Because of the importance of personal relationships and family in this population, Hispanics depend heavily on their social network, especially in times of need (e.g., chronic illness) (8). Multilevel support systems were linked to diabetes self-management in this sample. Specifically, support from all sources investigated in this study (i.e., self, family and friends, and neighborhood and community) was linked to better adherence to a diabetes self-care regimen (i.e., blood glucose monitoring, healthy diet, and exercise). Support for disease management also exhibited direct links with two physiological indicators. Specifically, individuals who reported receiving greater neighborhood and community support for disease management exhibited lower BMI and triglycerides than individuals who reported relatively less support. These findings suggest that culturally relevant approaches that include family and community may be effective in improving diabetes self-management and in reducing health risk among Hispanics with type 2 diabetes.

Several caveats should be considered in interpreting these findings. First, the cross-sectional design precludes inferences about causation. Although important associations were identified, additional (prospective) research is needed to determine whether increasing support for disease management consistently results in better diabetes self-management, less depression, and thus better health outcomes over time. Second, the results should be interpreted in the context of sample characteristics (i.e., the majority was female, had low income, and low U.S. acculturation). These characteristics limit the generalizability of the findings and prevent an examination of potential ethnic or cultural differences. Further, because the sample was relatively homogeneous, it was not possible to investigate gender, socioeconomic status, and acculturation as potential moderators of these associations. Additional research is needed to examine whether the links among support for disease management, diabetes self-management, depression, and clinical indicators of health risk persist outside the Hispanic population. 
Despite these limitations, this study showed that greater support for disease management from a variety of sources is linked to less depression, better diabetes self-management, and lower health risk among Hispanics with type 2 diabetes in the San Diego County, California, region. The need to identify culturally relevant approaches to improve health outcomes in this population is imperative. Interventions are especially needed in the U.S.-Mexico border region, an area that is characterized by disproportionate rates of type 2 diabetes and depression, poorer quality of care, and worse health outcomes. To improve health outcomes, future programs targeting diabetes selfmanagement among Hispanics residing in the U.S.-Mexico border region should

1. Cowie CC, Rust KF, Byrd-Holt DD, Gregg EW, Ford ES, Geiss LS, et al. Prevalence of diabetes and high risk for diabetes using A1C criteria in the U.S. population in 1988-2006. Diabetes Care. 2010;33(3):562-8.

2. Black SA. Diabetes, diversity, and disparity: what do we do with the evidence? Am J Public Health. 2002;92(4):543-8.

3. West SK, Klein R, Rodríguez J, Muñoz B, Broman AT, Sánchez R, et al. Diabetes and diabetic retinopathy in a Mexican-American population: Proyecto VER. Diabetes Care. 2001;24(7):1204-9.

4. Anders RL, Olson T, Wiebe J, Bean NH, DiGregorio R, Guillermina M, et al. Diabetes prevalence and treatment adherence in residents living in a colonia located on the West Texas, USA/Mexico border. Nurs Health Sci. 2008;10(3):195-202.

5. Spencer MS, Kieffer EC, Sinco BR, Palmisano G, Guzman JR, James SA, et al. Diabetes-specific emotional distress among African Americans and Hispanics with type 2 diabetes. J Health Care Poor Underserved. 2006;17(2 Suppl):88-105. assume a social-ecological perspective that considers multilevel influences on health and behavior. Specifically, interventions must address socioeconomic barriers, cultural attitudes, values, and beliefs that affect the ability and willingness of an individual to access care and modify behaviors.

Enhancing social-environmental support for disease management represents an avenue by which diabetes selfmanagement, emotional well-being, and health outcomes can be improved. Accordingly, consideration should be given to holding community-based health interventions and encouraging patients to invite family and friends to selfmanagement classes (11). Given the strong cultural emphasis on personal re-

\section{REFERENCES}

6. Mier N, Bocanegra-Alonso A, Zhan D, Wang S, Stoltz SM, Acosta-González RI, et al. Clinical depressive symptoms and diabetes in a binational border population. J Am Board Fam Med. 2008;21(3):223-33.

7. Misra R, Lager J. Ethnic and gender differences in psychosocial factors, glycemic control, and quality of life among adult type 2 diabetic patients. J Diabetes Complications. 2009;23(1):54-64.

8. Marin G, Marin BV. Research with Hispanic populations. Newbury Park, CA: Sage; 1991.

9. Egede LE, Osborn CY. Role of motivation in the relationship between depression, self-care, and glycemic control in adults with type 2 diabetes. Diabetes Educ. 2010; 36(2):276-83.

10. Pineda Olvera AE, Stewart SM, Galindo L, Stephens J. Diabetes, depression, and metabolic control in Latinas. Cultur Divers Ethnic Minor Psychol. 2007;13(3):225-31.

11. Weiler DM, Crist JD. Diabetes selfmanagement in a Latino social environment. Diabetes Educ. 2009;35(2):285-92. lationships and family connectedness among Hispanics, interventions to improve glycemic control and other health outcomes should recognize the strong influence natural support systems have on patients' implementation of diabetes self-management strategies. Socialenvironmental support for disease management may also represent a mechanism that, once mobilized, helps to sustain the effects of an intervention long after treatment has ended.

Acknowledgment. This work was supported by grant 1 R18 DK070666-01A1 from the National Institutes of HealthNational Institute of Diabetes and Digestive and Kidney Diseases awarded to A.P.-T.
12. Glasgow RE, Strycker LA, Toobert DJ, Eakin E. A social-ecologic approach to assessing support for disease selfmanagement: the Chronic Illness Resources Survey. J Behav Med. 2000;23(6): 559-83.

13. Toobert DJ, Hampson SE, Glasgow RE. The summary of diabetes self-care activities measure: results from 7 studies and a revised scale. Diabetes Care. 2000;23(7): 943-50.

14. Kroenke K, Spitzer RL, Williams JB. The PHQ-9: validity of a brief depression severity measure. J Gen Intern Med. 2001; 16(9):606-13.

15. Harris MI. Racial and ethnic differences in health care access and health outcomes for adults with type 2 diabetes. Diabetes Care. 2001;24(3):454-9.

Manuscript received on 17 May 2010. Revised version accepted for publication on 17 August 2010.

RESUMEN En este estudio se utilizó un marco socioecológico para analizar los factores predictivos de la depresión, la autogestión de la diabetes y los indicadores clínicos de riesgo para la salud en hispanos que padecen diabetes tipo 2 residentes en la zona fronteriza entre México y los Estados Unidos del Condado de San Diego en California. Se observaron vínculos importantes entre un mayor apoyo socioambiental para el manejo de la enfermedad y una presencia menor de la depresión, una mejor autogestión de la diabetes, y menores índices de masa corporal y concentraciones de triglicéridos séricos. La presencia menor de síntomas depresivos también se relacionó con niveles inferiores de hemoglobina A1c. Estos resultados indican que los programas dirigidos a mejorar la autogestión de la diabetes y los resultados en materia de salud en los hispanos que padecen diabetes tipo 2 deben tener en cuenta las influencias sociales y ambientales sobre la salud, el comportamiento y el bienestar emocional.

Palabras clave Hispanoamericanos; salud fronteriza; diabetes mellitus tipo 2; manejo de la enfermedad; depresión. 